\title{
Contribution of Pharmacogenetic Testing to Modeled Medication Change Recommendations in a Long-Term Care Population with Polypharmacy
}

\author{
Elaine A. Sugarman ${ }^{1} \cdot$ Ali Cullors $^{1} \cdot$ Joel Centeno $^{1} \cdot$ David Taylor $^{1}$
}

Published online: 8 November 2016

(c) The Author(s) 2016. This article is published with open access at Springerlink.com

\begin{abstract}
Background Among long-term care facility residents, polypharmacy is common, and often appropriate, given the need to treat multiple, complex, chronic conditions. Polypharmacy has, however, been associated with increased healthcare costs, adverse drug events, and drug interactions. The current study evaluates the potential medication cost savings of adding personalized pharmacogenetic information to traditional medication management strategies.

Methods One hundred and twelve long-term care residents completed pharmacogenetic testing for targeted variants in the following genes: CYP1A2, CYP2C9, CYP2C19, CYP2D6, CYP3A4/CYP3A5, HTR2A, HTR2C, SLC6A4, SLC6A2 COMT, OPRM1, SLCOIB1, VKORC1 and MTHFR. Following reporting of the IDgenetix Polypharmacy ${ }^{\circledR}$ test results, an internal medication management assessment was performed by a licensed clinical pharmacist to identify potential opportunities for regimen optimization through medication changes or discontinuations. The medication cost differences before and after the pharmacogenetic-guided review were assessed.

Results Medication review following pharmacogenetic result reporting identified 54 patients $(48.2 \%)$ with a total of 132 drug change recommendations (45 reductions; 87 replacements) and an average of 2.4 proposed medication changes (range 1-6) per patient. Medication cost savings related to the identified reduction and replacement opportunities exceeded the cost of testing and are estimated to be US $\$ 1300$ (year 2016 cost) per patient annually assuming full implementation.
\end{abstract}

\footnotetext{
David Taylor

dtaylor@altheadx.com

1 AltheaDx, San Diego, CA, USA
}

Conclusion Compared with traditional medication review, pharmacogenetic testing resulted in a $38 \%$ increase in the number of patients with current medication change opportunities and also offered valuable genetic information that could be referenced to personalize future prescribing decisions for all patients.

\section{Key Points}

When IDgenetix ${ }^{\circledR}$ Polypharmacy test results were added to a clinical pharmacist-guided medication review for long-term care polypharmacy patients, there was a $38 \%$ increase in patients identified with medication change opportunities, and the modeled drug cost savings exceeded testing costs within the first year.

Pharmacogenetic testing informed clinical prescribing recommendations for the majority of patients with current medication change recommendations, and provided personalized information that could be referenced in the medical record to inform future prescribing decisions.

\section{Introduction}

Polypharmacy is the concurrent use of multiple medications by a patient. Results of the 2004 National Nursing Home Survey found the prevalence of polypharmacy (defined as use of $\geq 9$ medications) among nursing home residents to be approximately $40 \%$ [1]. While the presence 
of multimorbidity for many long-term care facility patients may necessitate polypharmacy, careful medication monitoring is essential as excessive polypharmacy or inclusion of inappropriate medications may leave patients at risk for drug interactions and adverse drug events (ADEs).

Polypharmacy is often associated with an increase in unnecessary healthcare utilization due to drug interactions and ADEs, and associated falls, medication non-adherence, functional decline, and cognitive impairment, which result in increases in healthcare costs [2]. Nguyen et al. reported the incidence of adverse drug reactions among patients taking nine or more medications to be 2.3 times higher than among those patients taking fewer [3]; while a prospective cohort study by Doan and colleagues found a $50 \%$ risk of cytochrome enzyme-mediated drug-drug interactions among older hospitalized adults taking between five and nine medications and an increased risk of $100 \%$ among patients taking $>20$ medications [4].

Several studies have looked at strategies to make informed decisions to identify and reduce medications that are involved in drug-drug or drug-disease interactions, are potentially inappropriate in older adults, or may no longer be useful. To identify medication targets for reduction, such studies have utilized traditional resources such as drug-drug interaction identification tools, Beers criteria of potentially inappropriate medications, and drug indication documentation [5-7]. Today's medication therapy management utilizes a trial-and-error approach. A patient may be prescribed multiple drugs, at various dosages, and over extended periods of time before achieving the desired therapeutic effect. New tools such as pharmacogenetic (PGx) testing allow physicians to further personalize their patient's medication review. Utilizing the patient's DNA, PGx testing identifies how individuals may respond to drugs based on their unique genetic profile. This can help healthcare providers determine the right medication and the right dosage for their patients in a timely manner.

The US Food and Drug Administration (FDA) maintains a list of FDA-approved drugs and genes (currently 164 drug-gene pairs) with PGx information in their labeling, and notes that PGx can play an important role in avoiding adverse events, identifying responders and non-responders to medications, and optimizing drug dosage [8]. The Clinical Pharmacogenetics Implementation Consortium (CPIC) curates a list of 199 drug-gene pairs, of which 70 have FDA PGx label information where genetic testing is required, recommended, or considered 'actionable'. Forty of the drug-gene pairs are addressed in peer-reviewed guidelines published by the expert members of CPIC to support the "translation of genetic laboratory results into actionable prescribing decisions for specific drugs." [9].

Examples of drugs frequently prescribed in the longterm care (LTC) population and having FDA label and/or
CPIC guidance include selective serotonin reuptake inhibitors (SSRIs) (citalopram, escitalopram, sertraline, paroxetine, fluvoxamine), codeine, clopidogrel, and warfarin. The PGx guidance is based on cytochrome P450 (CYP450)-mediated metabolism (pharmacokinetics) for these medications and the potential for genetic variants in the CYP450 genes to alter the enzyme function and increase or decrease drug metabolism. For example, approximately $11 \%$ of Caucasian and $33 \%$ African American patients have been reported to have a deletion of both copies of their CYP2D6 gene, referred to as CYP2D6 $* 5$ (CYP2D6 $* 5 / * 5$ genotype) [10]. This results in a loss of CYP2D6 function and a 'poor metabolizer' phenotype. The CPIC guideline for 'CYP2D6 and CYP2C19 Genotypes and Dosing of Selective Serotonin Reuptake Inhibitors' notes that CYP2D6 poor metabolizers should be prescribed an alternative to paroxetine and fluvoxamine, or if use is warranted, reduce dose by $50 \%$ (paroxetine) or $25-50 \%$ (fluvoxamine) [11]. Similarly, CPIC guidelines recommend that CYP2D6 poor metabolizers avoid use of codeine and tramadol and exercise caution in using hydrocodone and oxycodone since the metabolism of each of these analgesics involves CYP2D6 [12].

The utility of integrating PGx information into prescribing decisions has been assessed in a variety of settings. Schildcrout et al. evaluated the frequency with which 56 medications known to be impacted by genetic variants were prescribed among 52,942 medical home patients over a 5-year period, and determined that $65 \%$ of the cohort would be exposed to at least one PGx-influenced medication, and $>10 \%$ would be exposed to at least four [13]. Several studies have evaluated the benefit of utilizing PGx information prior to making prescribing decisions to avoid ADEs and improve medication cost savings [13, 14], while others have evaluated the use of PGx information to support medication changes following treatment failure and ADEs, or to achieve reduction in polypharmacy $[15,16]$.

The aim of this study was to evaluate the medication cost impact of pharmacist-guided, PGx-informed, regimen review in patients receiving multiple medications in a longterm care setting.

\section{Methods}

Samples were received for testing from patients residing in one healthcare organization consisting of three LTC facilities. Medication lists reflecting patient regimens (including prescriptions, over-the-counter [OTC] medications, and supplements) were submitted to the laboratory along with a test order and the collected buccal swab. This material and documentation provided the baseline information for number of medications and dosages. All 
samples were processed in accordance with the Centers for Medicare and Medicaid Services (CMS) regulations in a College of American Pathologists (CAP)-accredited, Clinical Laboratory Improvement Amendments (CLIA)certified laboratory (AltheaDx, CA, USA). Using cheek swabs, buccal epithelial samples were collected and shipped to the laboratory at room temperature, together with test requisition forms and supporting documentation. Genomic DNA was extracted (QIAGEN DSP DNA Midi Kit, QIAgen, CA, USA) and quantified using a Nanodrop 8000 spectrophotometer (ThermoFisher Scientific, Waltham, MA, USA). Genotyping was performed using the TaqMan OpenArray platform (ThermoFisher, CA, USA) and CYP2D6 copy number variation was performed using TaqMan real-time PCR (ThermoFisher, CA, USA) to identify targeted PGx variants in 15 genes: CYP1A2, CYP2C9, CYP2C19, CYP2D6, CYP3A4/CYP3A5, HTR2A, HTR2C, SLC6A4, SLC6A2 COMT, OPRM1, SLCO1B1, VKORC1, and MTHFR. Variants tested in each of the genes were found at approximately $\geq 1 \%$ in the US population. Allele frequencies were monitored longitudinally for quality control purposes.

Drug-gene and drug-drug interactions were identified and characterized from an extensive review of FDA-approved package inserts, professional PGx guidelines (e.g., CPIC), and primary scientific literature. This information is currently utilized in the proprietary IDgenetix ${ }^{\circledR}$ Polypharmacy algorithm which was validated in accordance with CLIA and Clinical and Laboratory Standards Institute (CLSI) requirements. Results of genetic testing were processed through the proprietary IDgenetix ${ }^{\circledR}$ Polypharmacy algorithm, evaluating the patient's genetic results and CYPmediated drug interactions with concomitant medications, herbal supplements or other dietary or environmental factors, to provide personalized result interpretations. IDgenetix ${ }^{\circledR}$ Polypharmacy results were provided back to the referring physicians via reports which listed commonly used medications grouped by indication and categorized as 'use as directed' or 'use with caution and/or increased monitoring' based on patient results (Fig. 1).

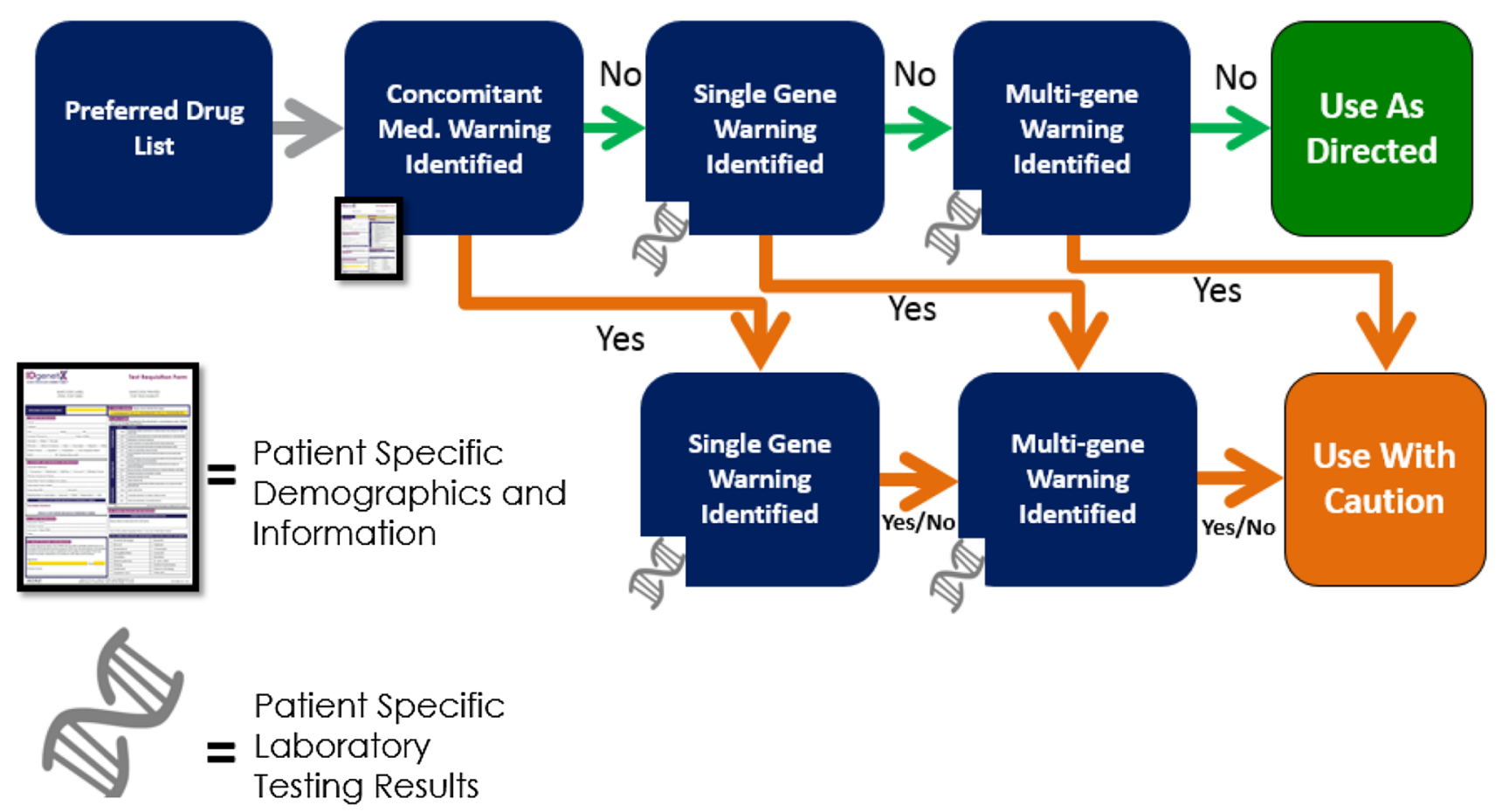

Fig. 1 IDgenetix ${ }^{\circledR}$ algorithm overview. The IDgenetix ${ }^{\circledR}$ test uses a proprietary algorithm to assess the appropriateness of a given medication for a specific patient. (1) The process begins by the physician providing a current medication list for the patient, termed the 'Preferred Drug List'. (2) The algorithm first identifies any adverse drug-drug interactions through the 'Concomitant Medication Warning Filter'. (3) The algorithm then uses the genetic information derived from the buccal swab to characterize any gene-drug interactions using the 'Single Gene Filter'. (4) Lastly, individual gene-drug interactions are further cross-referenced against one another to characterize any complex relationships among genes in biochemical pathways in the 'Multi-Gene Filter'. If a specific medication does not have any drug-drug or drug-gene interactions identified in any of the three filters, it is classified as 'Use as Directed' (see green arrows). If any one of the three algorithmic filters identifies an issue with a specific drug, that compound is classified as 'Use with Caution' (see orange arrows), and the appropriate warnings are provided. The 'Use as Directed' and 'Use with Caution' classifications are then used by the physician for making an informed decision on medication therapy management 
Following reporting to the referring physician, an IDgenetix $^{\circledR}$ internal medication management assessment was performed by a licensed Doctor of Pharmacy (Pharm.D.) to identify potential opportunities for regimen optimization through medication changes or discontinuations implied by the PGx analysis. Other more traditional considerations influential to the proposed improvements included drug-drug and drug-disease state interaction evaluation and pill burden reduction through the use of combination products (regimen consolidation). To facilitate data analysis, the suggested changes were categorized according to their principle motivation as follows: 'genetics: pharmacokinetic (PK) gene', where a CYP gene variant predicts increased or decreased drug metabolism; 'genetics: pharmacodynamic (PD) gene', where a non-CYP gene variant impacts drug response; 'drug-drug interactions'; 'drug-disease state interactions'; and 'regimen consolidation', where an alternate medication with additional therapeutic benefit or a combination medication could reduce pill burden.

The predicted medication costs associated with the Pharm.D.-identified regimen modification opportunities were modeled. For each medication, the current dosage was determined from review of medication orders that accompanied the sample submission documentation. The average wholesale price (AWP) corresponding to the appropriate formulation was determined on a per-month basis, using per-pill pricing derived from UpToDate/Lexicomp [17]. A 16.4\% ingredient cost and US\$3.73 dispense fee was then applied to the initial AWP, per State of Florida guidelines in 'Medicaid Covered Outpatient Drug Reimbursement Information' for quarter ending December 2014 [18]. The net change in medication costs were calculated for the patients with recommended changes by adding the savings from drug reductions to the cost difference (increased or decreased) resulting from drug replacements accounting for the cost of testing for all patients (i.e. those with and without recommended changes). The overall cost savings were calculated over 1-, 2-, and 2.3-year (average length of study for nursing home patients [19]) timelines and cost differences were also assessed according to therapeutic drug class.

\section{Results}

Samples were received for testing from 116 patients and results were reported for 112 patients (two patients had their test order cancelled by the referring physician, one patient sample had insufficient DNA, and one patient sample failed due to the assay). Among the 112 reported patients, $64(57 \%)$ were female, 48 (43\%) were male, and the average age was 74.2 years (range 34-100). Review of detailed medication orders for each patient revealed patients to be taking an average of 19 (range 6-40) pharmacologically active substances combining prescription and OTC medications and herbal/vitamin supplements.

\subsection{Patients with Change Recommendations}

After Pharm.D. review, 132 drug change recommendations (45 reductions; 87 replacements) were identified for 54 patients (48.2\% of tested population), with an average of 2.4 medication changes (range 1-6) per patient. The bases for the recommended therapeutic modifications among the 54 patients are displayed in Table 1 and Fig. 2. A patient may have had one or more identified reasons for their medication changes. For instance, for 12 patients, change recommendations were noted for three reasons, genetics: PK gene (metabolizer status) related, genetics: PD gene related, and drug-drug interactions. PGx results were a factor in one or more modification recommendations for 94.4\% (51/54) of patients. Individually, altered metabolizer phenotype (PK), as determined by CYP gene variant status, impacted current medications for $79.6 \%$ (43/54) of patients, and PD gene variants were implicated for $61.1 \%$ (33/54).

As displayed in Table 1, 15 of the 54 patients had medication change reasons that were exclusively genetic (PK, PD, or both). This represents a 38.4\% (15/39) increase in patients identified with medication change opportunities as compared with traditional evaluations relying solely on drug-drug or drug-disease state interactions, which would have identified only 39 patients in the current analysis.

The overall cost savings within a 12-month period for the 112 patients in this study (including all drug classes evaluated) was calculated to be US\$145,664 (year 2016 cost). This translates to an estimated savings of approximately US\$1300 per patient on an annualized basis. Given that the average length of stay for a nursing home resident is 2.3 years [19], this translates to a cumulative savings of approximately US\$3000 per patient over their entire length of stay.

When predicted cost savings were evaluated by therapeutic treatment class, the greatest savings among drug reductions were seen for gastrointestinal and psychotropic drugs. Among drug replacements, psychotropic drugs had the greatest cost savings, followed by cardiovascular. Taken together, gastrointestinal, psychotropic, and cardiovascular drug reductions and replacements accounted for over US\$150,000 in annualized savings and accounted for $87 \%$ of the patients evaluated. The predominance of these therapeutic areas among the proposed interventions reflects the relatively larger contributions of these medical disciplines to the current PGx knowledge base. At present, practitioners utilizing these therapeutic classes could gain most from 
Table 1 Reasons for clinical pharmacist medication change recommendations. Drug classes evaluated included gastrointestinal, psychotropic, analgesic, cardiovascular, neurologic, endocrine, anticonvulsant, and musculoskeletal drugs

\begin{tabular}{|c|c|c|c|c|c|c|c|}
\hline $\begin{array}{l}\text { Genetics: } \\
\text { CYP450 } \\
\text { genes }^{\mathrm{a}}\end{array}$ & $\begin{array}{l}\text { Genetics: } \\
\text { non-CYP450 } \\
\text { genes }^{\mathrm{b}}\end{array}$ & $\begin{array}{l}\text { Drug-drug } \\
\text { interactions }\end{array}$ & $\begin{array}{l}\text { Regimen } \\
\text { consolidation }\end{array}$ & $\begin{array}{l}\text { Drug-disease } \\
\text { state interaction }\end{array}$ & $\begin{array}{l}\text { Number } \\
\text { of patients }\end{array}$ & $\begin{array}{l}\text { Total number } \\
\text { of drugs } \\
\text { reduced }\end{array}$ & $\begin{array}{l}\text { Total number of } \\
\text { current drugs to } \\
\text { be replaced }\end{array}$ \\
\hline$X$ & $X$ & & & & 7 & 5 & 10 \\
\hline \multirow[t]{2}{*}{$X$} & & & & & 5 & 2 & 6 \\
\hline & $X$ & & & & 3 & 3 & 3 \\
\hline \multirow[t]{2}{*}{$X$} & $X$ & $X$ & & & 12 & 10 & 20 \\
\hline & $X$ & $X$ & & & 5 & 4 & 6 \\
\hline$X$ & & $X$ & & & 5 & 3 & 8 \\
\hline$X$ & $X$ & $X$ & $X$ & & 3 & 5 & 6 \\
\hline $\mathrm{X}$ & $X$ & & $X$ & & 2 & 2 & 5 \\
\hline$X$ & & $X$ & & $\mathrm{X}$ & 2 & 2 & 4 \\
\hline \multirow[t]{4}{*}{$X$} & & $X$ & $X$ & & 2 & 3 & 5 \\
\hline & & $X$ & $X$ & & 2 & 3 & 4 \\
\hline & & $X$ & & & 1 & 0 & 1 \\
\hline & & $X$ & & $\mathrm{X}$ & 1 & 0 & 1 \\
\hline X & & $X$ & $X$ & $\mathrm{X}$ & 1 & 1 & 2 \\
\hline$X$ & & & $X$ & $\mathrm{X}$ & 1 & 1 & 3 \\
\hline \multirow[t]{3}{*}{$X$} & $X$ & & & $X$ & 1 & 0 & 2 \\
\hline & & & $X$ & & 1 & 1 & 1 \\
\hline & & & & Total & 54 & 45 & 87 \\
\hline
\end{tabular}

a $C Y P 1 A 2, C Y P 2 D 6, C Y P 2 C 9, C Y P 2 C 19, C Y P 3 A 4$

b COMT, OPRM1, SLCO1B1, VKORC1, MTHFR

Fig. 2 Reasons for medication change recommendations

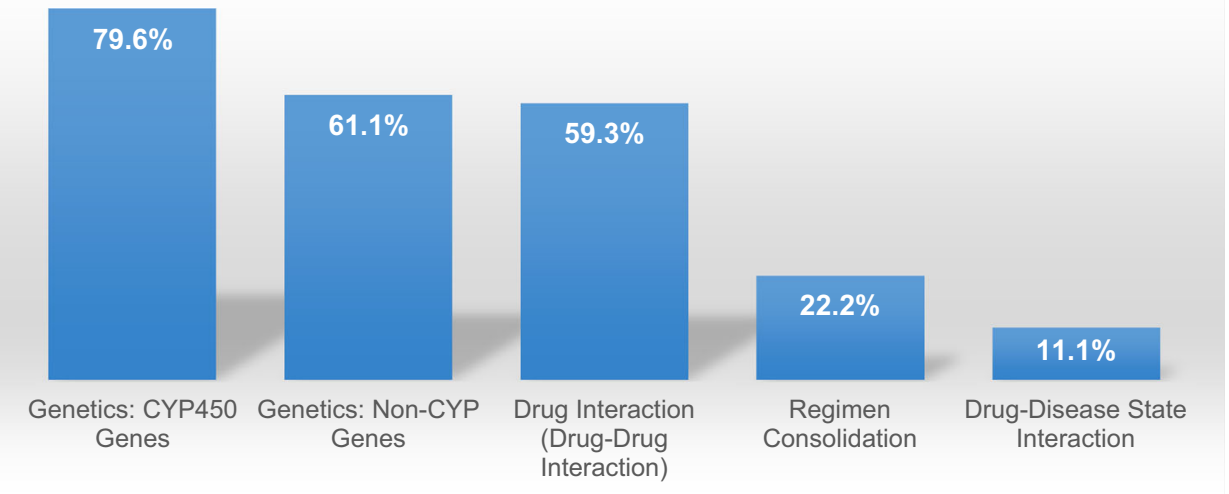

adopting this approach. However, PGx therapeutic applicability can only broaden as the range and scope of validated clinically relevant PGx associations expands.

\subsection{Patients without Change Recommendations}

When the same criteria for potential change recommendations (genetics: PK, genetics: PD, drug-drug interaction, regimen consolidation, and drug-disease interaction) were applied to all 112 patients, no change recommendations were made for 58. Among the 58 patients for whom no changes to their current regimen were identified, the average age was 76.6 years and the average number of current medications was 16.8 (range 6-35). We assessed the frequency of select genetic variants that could impact clinician decision making if future medication changes were to be considered. These findings are displayed in Table 2. 
Table 2 Frequency of select metabolic and genetic alterations among patients without current regimen change recommendations

\begin{tabular}{|c|c|c|c|}
\hline Gene variant & Count & $\begin{array}{l}\text { Frequency among patients } \\
\text { without current medication } \\
\text { change recommendations }(\%)\end{array}$ & $\begin{array}{l}\text { Example medications warranting } \\
\text { dosage adjustment or caution }\end{array}$ \\
\hline CYP2C19-altered metabolism (PM or UM) & 19 & 32.8 & $\begin{array}{l}\text { Tricyclic antidepressants, citalopram, } \\
\text { escitalopram, clopidogrel }\end{array}$ \\
\hline CYP2D6 altered metabolism (UM or IM) & 2 & 3.5 & Codeine, hydrocodone \\
\hline $\begin{array}{l}\text { CYP2C9 (reduced function) plus VKORC1 } \\
(\text { (c. }-1639 \mathrm{G}>\mathrm{A} A / \mathrm{G} \text { or } \mathrm{A} / \mathrm{A})\end{array}$ & 10 & 17.2 & Warfarin \\
\hline SLCO1B1 $(\mathrm{c} .521 \mathrm{~T}>\mathrm{C} \mathrm{C} / \mathrm{T}$ or $\mathrm{C} / \mathrm{C})$ & 15 & 25.9 & Simvastatin \\
\hline OPRM1 (c.118 A $>\mathrm{G} \mathrm{A} / \mathrm{G}$ or $\mathrm{G} / \mathrm{G})$ & 12 & 20.7 & Morphine, hydrocodone, other opioids \\
\hline $\operatorname{HTR} 2 A(\mathrm{c} .998 \mathrm{G}>\mathrm{A} \mathrm{G} / \mathrm{G}$ or $\mathrm{A} / \mathrm{A})$ & 28 & 48.3 & SSRIs \\
\hline
\end{tabular}

IM intermediate metabolizer, PM poor metabolizer, SSRIs selective serotonin reuptake inhibitors, UM ultrarapid metabolizer

\section{Discussion}

Previous studies of pharmacist or physician medication management review have focused on drug-drug interactions or potentially inappropriate medications $[5,6]$. In the current study, we add another valuable, personalized, patient variable to the assessment: PGx. Patient medications were identified as candidates for reduction or replacement based on PGx findings, drug-drug interactions, drug-disease interactions or opportunities for regimen consolidation.

Additional insight from genetic analysis can inform selection of medications for reduction or replacement, and, in combination with drug interaction information, identify patients for whom the impact of combined drug-drug interaction and genetics (phenoconversion) poses a greater risk than would be recognized were either considered in isolation. Genetic analysis can guide both medication prescribing and deprescribing as well as enhance drugdrug interaction evaluation by informing the impact of metabolic inducers and inhibitors. To illustrate, consider the example case of a 79-year-old male whose extensive regimen included amiodarone, paroxetine, spironolactone, tamsulosin, warfarin, lorazepam, tramadol, meloxicam, zolpidem, insulin, digoxin, alendronate, bumetanide, and levothyroxine. Genetic analysis revealed variant results predictive of CYP2D6 poor metabolizer (PM) and CYP2C9 intermediate metabolizer (IM) phenotypes. As several of the patient's medications are CYP2D6 (paroxetine, tamsulosin, tramadol) and CYP2C9 (warfarin, meloxicam) substrates, knowledge of the patient's altered metabolizer status provides valuable insight for safer medication selection. For paroxetine, CYP2D6 PM status increases the likelihood of adversity due to greater systemic exposure, while its antiplatelet and osteopenic effects threaten to exacerbate comorbidities. The combination of CYP2D6 PM paired with CYP3A4 inhibition from amiodarone poses risks for tramadol (decreased efficacy, increased adversity) and tamsulosin (increased risk of hypotension, orthostasis, and syncope). Both paroxetine and tramadol are included on the FDA Pharmacogenomic Biomarkers in Drug Labeling list for CYP2D6 poor metabolizer status and have CPIC guidelines noting caution for use in 2D6 PMs. Similarly, warfarin appears on the FDA Biomarker list and in CPIC guidelines for CYP2C9 reduced function. This patient's CYP2C9 IM result in combination with amiodarone CYP2C9 inhibition leads to increased systemic exposure and sensitivity to minor changes in dosage, suggesting that a switch to a directacting oral anticoagulant (apixaban, dabigatran, edoxaban or rivaroxaban), dosed appropriately for renal function, might be beneficial. Medication recommendations included replacement of tramadol with hydromorphone, replacement of paroxetine with mirtazapine, and reduction of zolpidem leading to an estimated annualized savings of US\$934.

Previous studies employing medication management review followed by implementation of recommended drug reductions have demonstrated substantial health and cost benefits. Garfinkel and colleagues implemented discontinuation of 332 different drugs among 119 study patients in a geriatric nursing ward without significant adverse effects. In comparison with a 71-patient control group, the study patients had significantly lower referral rates to acute care facilities, 1-year mortality rates, and drug costs [20].

Our study added a Pharm.D. review of PGx results to the traditional medication review resources. The inclusion of PGx criteria resulted in $38 \%$ more patients being identified with reduction/replacement opportunities than would have been identified on the basis of drug-drug, drug-disease or regimen consolidation opportunities alone. The goal was to use these factors to model optimization of patient medication regimens. As such, rather than focus solely on opportunities to eliminate medications, we sought to both 
eliminate potentially ineffective medications and to also replace medications with unfavorable profiles with those that could offer improved treatment options.

All 112 patients referred for testing were taking six or more medications at the time of testing. Of the 112 patients, 107 (95.5\%) were taking $\geq 10$ medications (including prescription and OTC medications). It is unclear whether this high frequency reflects patient selection for PGx testing from these nursing home facilities or is reflective of the larger resident population. Previous studies of polypharmacy (defined as nine or more medications) among nursing home residents have reported rates of 39.7 and $46.3 \%$ [1,6], while an earlier, unpublished analysis of polypharmacy among $>37,000$ samples referred to our lab for clinical PGx testing identified $44 \%$ of patients having more than nine medications. Given the multiple morbidities and clinical complexity of most nursing home residents, use of multiple medications is not unexpected. With research supporting a strong association between polypharmacy and increased risk for adverse drug events, drug interactions, medication non-adherence, and falls, medication management strategies aimed at reducing unnecessary or genetically incompatible medications are expected to benefit patient health while reducing costs $[2,6,21]$.

While our analysis focused on medication cost changes related to proposed regimen modifications in the 54 patients deemed upon Pharm.D. review to potentially benefit from reductions or replacements to their current regimen, PGx results were obtained for all 112 patients. PGx information in the patient medical record is of clinical value in informing future prescribing decisions for all patients. Among the patients without current change recommendations, for example, $22 \%$ carry a c. 118 A $>\mathrm{G}$ variant in the OPRMI gene that has been associated with higher sensitivity to pain, higher opioid dosage requirement to achieve analgesic effect, and unchanged or lower risk for opioid-related side effects [22]. As nursing home clinicians adjust patient medications in response to changing needs for pain control, knowledge of a patient's OPRM1 c.118A $>$ G status can help inform medication selection and dosage decisions.

Our analysis modeled medication cost savings related to the Pharm.D.-recommended drug reductions and replacements in a polypharmacy nursing home population and estimated cost savings of US\$1300, US $\$ 2600$, and US $\$ 3000$ per patient at 1,2 , and 2.3 years, respectively. Previous studies of pharmacist [5] or physician [6] interventions on medical management of a long-term care population have reported 80 and $86 \%$ acceptance of interventions by the primary attending physician, while Brixner et al., evaluating a clinical decision support tool in a $>65$-year-old cohort, found providers followed $46 \%$ of test recommendations [16]. If we apply a conservative $70 \%$ acceptance rate to our recommendations, the resulting annual medication cost savings of US $\$ 910.40$ exceeds the cost of PGx testing in the first year. As studies of medication review and reduction in polypharmacy nursing home patients have previously demonstrated $[6,20,23]$, we would expect implementation of our medical management strategy to conservatively yield not only medication cost savings but also potential additional healthcare cost savings resulting from reductions in adverse drug events, hospitalizations, falls, and medication administration costs. A similar cost analysis was performed by Saldivar et al. [15] using the IDgenetix ${ }^{\circledR}$ Polypharmacy test demonstrating annual savings of US\$621 per patient. The primary drug class that contributed the most cost savings in that study was psychotropic drugs. In contrast, the greatest savings in our study were mostly attributed to gastrointestinal medications. Additionally, our study resulted in greater cost savings (US\$1300 vs US\$621 on an annual basis), indicating that the IDgenetix ${ }^{\circledR}$ Polypharmacy test is capable of generating a range of savings from a variety of different drug classes.

While numerous studies have evaluated methods and benefits of medication management review and deprescribing in long-term care facilities, here we illustrate the potential impact of adding personalized PGx information to the medication review process. Among patients with recommended drug reductions or replacements, PGx results were a contributing factor in one or more recommended changes in $94 \%$, and resulted in a $38 \%$ increase in the number of patients identified with medication change recommendations than would be recognized by traditional review criteria. These are promising results, yet limitations present in this study should be addressed in future efforts. Our analysis was based on independent review of regimen information by a single clinical pharmacist, and medication change recommendations were not communicated and implemented, therefore acceptance rate and follow-up monitoring for efficacy and side-effect profiles could not be established. Our cost analysis was a simple pre- versus post-PGx and projected cost savings based on a subset of patients tested and was not compared with a control group. In future studies, a more formal economic analysis can be done to account for adverse drug reactions and the associated medical costs along with projected medication savings from PGx testing, utilizing a control and experimental group.

Future studies evaluating the integration of PGx test results into medication management review (including physician alerts through an electronic medical record system), implementation of recommendations, and resulting healthcare and cost benefits will help assess the great 
potential for continually improving patient care with a truly personalized medicine approach.

\section{Compliance with Ethical Standards}

Conflict of interest Elaine A. Sugarman was an employee of AltheaDx at the time the study was conducted. All other authors (Ali Cullors, Joel Centeno, and David Taylor) are full-time employees and stockholders at AltheaDx.

Funding This study was funded by AltheaDx, Inc.

Ethical approval and informed consent Patients were consented by the healthcare provider (HCP) in accordance with local and state laws. Every patient provided consent for pharmacogenetic testing as part of CLIA requirements. Informed consent, approved by an Institutional Review Board (IRB), was not required for this study.

Open Access This article is distributed under the terms of the Creative Commons Attribution-NonCommercial 4.0 International License (http://creativecommons.org/licenses/by-nc/4.0/), which permits any noncommercial use, distribution, and reproduction in any medium, provided you give appropriate credit to the original author(s) and the source, provide a link to the Creative Commons license, and indicate if changes were made.

\section{References}

1. Dwyer LL, Han B, Woodwell DA, Rechtsteiner EA. Polypharmacy in nursing home residents in the United States: results of the 2004 National Nursing Home Survey. Am J Geriatr Pharmacother. 2010;8(1):63-72.

2. Maher RL, Hanlon JT, Hajjar ER. Clinical consequences of polypharmacy in elderly. Expert Opin Drug Safety. 2014;. doi:10. 1517/14740338.2013.827660.

3. Nguyen JK, Fouts MM, Kotabe SE, Lo E. Polypharmacy as a risk factor for adverse drug reactions in geriatric nursing home residents. Am J Geriatr Pharmacother. 2006;4(1):36-41 (PubMed PMID: 16730619).

4. Doan J, Zakrzewski-Jakubiak H, Roy J, Turgeon J, Tannenbaum C. Prevalence and risk of potential cytochrome P450-mediated drug-drug interactions in older hospitalized patients with polypharmacy. Ann Pharmacother. 2013;47(3):324-32.

5. Mestres C, Hernandez M, Llagostera B, Espier M, Chandre M. Improvement of pharmacological treatments in nursing homes: medication review by consultant pharmacists. Eur J Hosp Pharm. 2015;22(4):207-11.

6. Kojima G, Bell C, Tamura B, Inaba M, Lubimir K, Blanchette PL, Iwasaki W, Masaki K. Reducing cost by reducing polypharmacy: the polypharmacy outcomes project. J Am Med Dir Assoc. 2012;13(9):818.e11-5.

7. Scott IA, Hilmer SN, Reeve E, Potter K, Le Couteur D, Rigby D, Gnjidic D, Del Mar CB, Roughead EE, Page A, Jansen J, Martin JH. Reducing inappropriate polypharmacy: the process of deprescribing. JAMA Intern Med. 2015;175(5):827-34.

8. Table of Pharmacogenomic Biomarkers in Drug Labeling. In: FDA U.S. Food and Drug Administration. 2015. http://www.fda. gov/drugs/scienceresearch/researchareas/pharmacogenetics/ucm08 3378.htm. Accessed 4 Apr 2016.

9. https://cpicpgx.org/. Accessed April 5, 2016.
10. Beoris M, Amos Wilson J, Garces JA, Lukowiak AA. CYP2D6 copy number distribution in the US population. Pharmacogenet Genomics. 2016;26(2):96-9.

11. Hicks JK, Bishop JR, Sangkuhl K, Muller DJ, Ji Y, Leckband SG, Leeder JS, Graham RL, Chiulli DL, LLerena A, Skaar TC, Scott SA, Stingl JC, Klein TE, Caudle KE, Gaedigk A. Clinical Pharmacogenetics Implementation Consortium (CPIC). Guideline for CYP2D6 and CYP2C19 genotypes and dosing of selective serotonin reuptake inhibitors. Clin Pharmacol Ther. 2015;98(2):127-34.

12. Crews KR, Gaedigk A, Dunnenberger HM, Leeder JS, Klein TE, Caudle KE, Haidar CE, Shen DD, Callaghan JT, Sadhasivam S, Prows CA, Kharasch ED, Skaar TC. Clinical Pharmacogenetics Implementation Consortium (CPIC). Guideline for cytochrome P450 2D6 genotype and codeine therapy: 2014 update. Clin Pharmacol Ther. 2014;95(4):376-82.

13. Schildcrout JS, Denny JC, Bowton E, Gregg W, Pulley JM, Basford MA, Cowan JD, Xu H, Ramirez AH, Crawford DC, Ritchie MD, Peterson JF, Masys DR, Wilke RA, Roden DM. Optimizing drug outcomes through pharmacogenetics: a case for preemptive genotyping. Clin Pharmacol Ther. 2012;92(2):235-42.

14. Van Driest SL, Shi Y, Bowton EA, Schildcrout JS, Peterson JF, Pulley J, Denny JC, Roden DM. Clinically actionable genotypes among 10,000 patients with preemptive pharmacogenomic testing. Clin Pharmacol Ther. 2014;95(4):423-31.

15. Saldivar JS, Taylor D, Sugarman EA, Cullors A, Garces JA, Oades K, Centeno J. Initial assessment of the benefits of implementing pharmacogenetics into the medical management of patients in a long-term care facility. Pharmgenomics Pers Med. 2016;19(9):1-6.

16. Brixner D, Biltaji E, Bress A, Unni S, Ye X, Mamiya T, Ashcraft $\mathrm{K}$, Biskupiak J. The effect of pharmacogenetic profiling with a clinical decision support tool on healthcare resource utilization and estimated costs in the elderly exposed to polypharmacy. J Med Econ. 2016;19(3):213-28.

17. Lexicomp. Contents: General drug information. In: UpToDate. https://www.uptodate.com/contents/table-of-contents/drug-infor mation/general-drug-information. Accessed 22 Apr 2016.

18. Medicaid Covered Outpatient Prescription Drug Reimbursement Information by State Quarter Ending December 2014. In: Medicaid.gov. 2014. https://www.medicaid.gov/medicaid-chip-programinformation/by-topics/benefits/prescription-drugs/state-prescriptiondrug-resources.html. Accessed 2 Feb 2015.

19. Jones AL, Dwyer LL, Bercovitz AR, Strahan GW. The National nursing home survey: 2004 overview. National center for health statistics. Vital Health Stat. 2009;13(167):1-155.

20. Garfinkel D, Zur-Gil S, Ben-Israel J. The war against polypharmacy: a new cost-effective geriatric-palliative approach for improving drug therapy in disabled elderly people. Isr Med Assoc J. 2007;9(6):430-4.

21. Marcolina ST, Winn P. Medication management in long-term care. In: Fenstemacher PA, Winn P, editors. Post-acute and longterm medicine, current clinical practice. Switzerland: Springer International Publishing; 2016. p. 307-26.

22. Mura E, Govoni S, Racchi M, Carossa V, Ranzani GN, Allegri M, van Schaik RH. Consequences of the $118 \mathrm{~A}>\mathrm{G}$ polymorphism in the OPRM1 gene: translation from bench to bedside? J Pain Res. 2013;6:331-53.

23. Alldred D, Zermansky A, Petty D, Raynor D, Freemantle N, Eastaugh J, Bowie P. Clinical medication review by a pharmacist of elderly people living in care homes: pharmacist interventions. Int J Pharm Prac. 2007;15(2):93-9. 